\title{
Multi-person Multi-attribute Decision Making Problems Based on Interval-valued Intuitionistic Fuzzy Information
}

\author{
Jin Han Park ${ }^{1}$, Young Chel Kwun ${ }^{2}$ and Mi Jung Son ${ }^{3}$ \\ 1 Department of Applied Mathematics, Pukyong National University, Pusan 608-737, Korea \\ 2 Department of Mathematics, Dong-A University, Pusan 604-714, Korea \\ 3 Department of Mathematics, Korea Maritime University, Pusan 606-791, Korea
}

\begin{abstract}
Based on the interval-valued intuitionistic fuzzy hybrid geometric (IIFHG) operator and the interval-valued intuitionistic fuzzy weighted geometric (IIFWG) operator, we investigate the group decision making problems in which all the information provided by the decision-makers is presented as interval-valued intuitionistic fuzzy decision matrices where each of the elements is characterized by interval-valued intuitionistic fuzzy numbers, and the information about attribute weights is partially known. A numerical example is used to illustrate the applicability of the proposed approach.
\end{abstract}

Key Words : Interval-valued intuitionistic fuzzy sets, IIFHG operator, IIFWG operator, score function, multi-person multi-attribute decision making.

\section{Introduction}

Interval-valued intuitionistic fuzzy sets, introduced by Atanassov and Gargov [1], each of which is characterized by a membership function and a non-membership function whose values are intervals rather than exact numbers, are a very useful means to describe the decision information in the process of decision making. Some researcher have applied the interval-valued intuitionistic fuzzy set theory to the field of decision making. Xu and Chen [15] developed some arithmetic aggregation operators, such as the intervalvalued intuitionistic fuzzy weighted averaging (IIFWA) operator, the interval-valued intuitionistic fuzzy ordered weighted averaging (IIFOWA) operator and the intervalvalued intuitionistic fuzzy hybrid averaging (IIFHA) operator for aggregating interval-valued intuitionistic fuzzy information, and gave an application of IIFHA operator to multi-attribute group decision making with interval-valued intuitionistic fuzzy information. Xu [13] developed some geometric aggregation operator, such as the interval-valued intuitionistic fuzzy geometric (IIFG) operator and intervalvalued intuitionistic fuzzy weighted geometric (IIFWG) operator and applied them to multi-attribute group decision making with interval-valued intuitionistic fuzzy information. $\mathrm{Xu}$ and Chen [14] and Wei and Wang [11], respectively, developed some geometric aggregation operator, such as the interval-valued intuitionistic fuzzy or-

Manuscript received Oct. 7, 2010; revised Nov. 17, 2010; Accepted Dec. 2, 2010.

Corresponding Author : Young Chel Kwun (yckwun@dau.ac.kr) This study was supported by research funds from Dong-A University. dered weighted geometric (IIFOWG) operator and intervalvalued intuitionistic fuzzy hybrid geometric (IIFHG) operator and applied them to multi-attribute group decision making with interval-valued intuitionistic fuzzy information. However, they used the IIFWOG operator and the IIFWG operator in the situation where the information about attribute weights is completely known.

In this paper, we study some properties of the IIFWG and IIFWOG operators and then investigate the group decision making problems in which all the information provided by the decision-makers is presented as intervalvalued intuitionistic fuzzy decision matrices where each of the elements is characterized by interval-valued intuitionistic fuzzy number, and the information about attribute weights is partially known. First, we use the IIFHG operator to aggregate all individual interval-valued intuitionistic fuzzy decision matrices provided by the decision-makers into the collective interval-valued intuitionistic fuzzy decision matrix, and then we use the score function to calculate the score of each attribute value and construct the score matrix of the collective interval-valued intuitionistic fuzzy decision matrix. Based on the score matrix and the given attribute weight information, we establish some optimization models to determine the weights of attributes, and then we use the obtained attribute weights and the IIFWG operator to fuse the interval-valued intuitionistic fuzzy information in the collective interval-valued intuitionistic fuzzy deci- 
sion matrix to get the overall interval-valued intuitionistic fuzzy values of alternatives by which the raking of all the given alternatives can be found. Finally, a numerical example is given to illustrate the applicability of the proposed method.

\section{Relations and Aggregation Operators}

\subsection{Basic Concepts and Relations}

Let a set $X$ be fixed and $D[0,1]$ be the set of all closed subintervals of the interval $[0,1]$. An interval-valued intuitionistic fuzzy set (IVIFS) $A$ in $X$ is an object having the form:

$$
A=\left\{\left\langle x, \mu_{A}(x), \nu_{A}(x)\right\rangle: x \in X\right\},
$$

where $\mu_{A}: X \rightarrow D[0,1], \nu_{A}: X \rightarrow D[0,1]$ with the condition $\sup \mu_{A}(x)+\sup \nu_{A}(x) \leq 1$ for any $x \in X$.

The intervals $\mu_{A}(x)$ and $\nu_{A}(x)$ denote, respectively, the degree of belongingness and the degree of nonbelongingness of the element $x$ to $A$. Then for each $x \in X$, $\mu_{A}(x)$ and $\nu_{A}(x)$ are closed intervals and their lower and upper end points are denoted by $\mu_{A L}(x), \mu_{A U}(x), \nu_{A L}(x)$ and $\nu_{A U}(x)$, respectively, and thus we can replace (1) with $A=\left\{\left\langle x,\left[\mu_{A L}(x), \mu_{A U}(x)\right],\left[\nu_{A L}(x), \nu_{A U}(x)\right]\right\rangle: x \in X\right\}$, where $0 \leq \mu_{A U}(x)+\nu_{A U}(x) \leq 1$ for any $x \in X$.

For each IVIFS $A$ in $X$, we call

$$
\begin{aligned}
\pi_{A}(x) & =1-\mu_{A}(x)-\nu_{A}(x) \\
& =\left[1-\mu_{A U}(x)-\nu_{A U}(x), 1-\mu_{A L}(x)-\nu_{A L}(x)\right]
\end{aligned}
$$

an intuitionistic fuzzy interval of $X$ in $A$.

For convenience, we call $\tilde{a}=\langle[a, b],[c, d]\rangle$ an intervalvalued intuitionistic fuzzy number (IVIFN) [13], where $[a, b] \subset[0,1],[c, d] \subset[0,1]$ and $b+d \leq 1$.

$\mathrm{Xu}$ [13] defined a score function $s$ to measure a IVIFN $\tilde{a}$ as follows:

$$
s(\tilde{a})=\frac{1}{2}(a-c+b-d),
$$

where $s(\tilde{a}) \in[-1,1]$. The larger the value of $s(\tilde{a})$, the higher the IVIFN $\tilde{a}$. Especially, if $s(\tilde{a})=1$, then $\tilde{a}=$ $\langle[1,1],[0,0]\rangle$, which is the largest IVIFN; if $s(\tilde{a})=-1$, then $\tilde{a}=\langle[0,0],[1,1]\rangle$, which is the smallest IVIFN.

Wei and Wang [11] defined an accuracy function $h$ to evaluate the accuracy degree of a IVIFN $\tilde{a}$ as follows:

$$
h(\tilde{a})=\frac{1}{2}(a+b+c+d),
$$

where $h(\tilde{a}) \in[0,1]$. The larger the value of $h(\tilde{a})$, the higher the degree of accuracy of the IVIFN $\tilde{a}$.
From (2), we define the hesitancy degree of the IVIFN $\tilde{a}=\langle[a, b],[c, d]\rangle$ as the midpoint of intuitionistic fuzzy interval of $\tilde{a}$, i.e.,

$$
\pi(\tilde{a})=\frac{1}{2}((1-a-c)+(1-b-d)) .
$$

Then we get the relation between the hesitancy degree and the accuracy degree of the IVIFN $\tilde{a}$

$$
\pi(\tilde{a})=\frac{1}{2}((1-a-c)+(1-b-d))=1-h(\tilde{a}),
$$

i.e.,

$$
\pi(\tilde{a})+h(\tilde{a})=1 .
$$

From (6), we know that the higher the accuracy degree $h(\tilde{a})$, the lower the hesitancy degree $\pi(\tilde{a})$.

Based on the score function and the accuracy function, $\mathrm{Xu}$ [13] defined a method to compare two IVIFNs as follows:

Definition 1. Let $\tilde{a}_{1}=\left\langle\left[a_{1}, b_{1}\right],\left[c_{1}, d_{1}\right]\right\rangle$ and $\tilde{a}_{2}=$ $\left\langle\left[a_{2}, b_{2}\right],\left[c_{2}, d_{2}\right]\right\rangle$ be two IVIFNs, $s\left(\tilde{a}_{1}\right)=\frac{1}{2}\left(a_{1}-c_{1}+\right.$ $\left.b_{1}-d_{1}\right)$ and $s\left(\tilde{a}_{2}\right)=\frac{1}{2}\left(a_{2}-c_{2}+b_{2}-d_{2}\right)$ be the score of $\tilde{a}_{1}$ and $\tilde{a}_{2}$, respectively, and $h\left(\tilde{a}_{1}\right)=\frac{1}{2}\left(a_{1}+b_{1}+c_{1}+d_{1}\right)$ and $h\left(\tilde{a}_{2}\right)=\frac{1}{2}\left(a_{2}+b_{2}+c_{2}+d_{2}\right)$ be the accuracy degree of $\tilde{a}_{1}$ and $\tilde{a}_{2}$, respectively; then:

- if $s\left(\tilde{a}_{1}\right)<s\left(\tilde{a}_{2}\right)$, then $\tilde{a}_{1}$ is smaller than $\tilde{a}_{2}$, denoted by $\tilde{a}_{1}<\tilde{a}_{2}$

- if $s\left(\tilde{a}_{1}\right)=s\left(\tilde{a}_{2}\right)$, then

1) if $h\left(\tilde{a}_{1}\right)=h\left(\tilde{a}_{2}\right)$, then $\tilde{a}_{1}$ and $\tilde{a}_{2}$ represent the same information which denotes indifference between $\tilde{a_{1}}$ and $\tilde{a_{2}}$, denoted by $\tilde{a_{1}} \sim \tilde{a_{2}}$;

2) if $h\left(\tilde{a}_{1}\right)<h\left(\tilde{a}_{2}\right)$, then $\tilde{a}_{1}$ is smaller than $\tilde{a}_{2}$, denoted by $\tilde{a}_{1}<\tilde{a}_{2}$.

Theorem 1. Let $\tilde{a}_{1}=\left\langle\left[a_{1}, b_{1}\right],\left[c_{1}, d_{1}\right]\right\rangle$ and $\tilde{a}_{2}=$ $\left\langle\left[a_{2}, b_{2}\right],\left[c_{2}, d_{2}\right]\right\rangle$ be two IVIFNs; then we have

$a_{1} \leq a_{2}, b_{1} \leq b_{2}, c_{1} \geq c_{2}$ and $d_{1} \geq d_{2} \Longrightarrow \tilde{a}_{1} \leq \tilde{a}_{2}$.

Proof. Since $s\left(\tilde{a}_{1}\right)=\frac{1}{2}\left(a_{1}-c_{1}+b_{1}-d_{1}\right), s\left(\tilde{a}_{2}\right)=$ $\frac{1}{2}\left(a_{2}-c_{2}+b_{2}-d_{2}\right), a_{1} \leq a_{2}, b_{1} \leq b_{2}, c_{1} \geq c_{2}$ and $d_{1} \geq d_{2}$, we have

$$
\begin{aligned}
s\left(\tilde{a}_{1}\right) & -s\left(\tilde{a}_{2}\right) \\
& =\frac{1}{2}\left(a_{1}-a_{2}\right)+\left(b_{1}-b_{2}\right)+\left(c_{2}-c_{1}\right)+\left(d_{2}-d_{1}\right) .
\end{aligned}
$$

If $a_{1}=a_{2}, b_{1}=b_{2}, c_{1}=c_{2}$ and $d_{1}=d_{2}$, then $\tilde{a}_{1}=\tilde{a}_{2}$; otherwise, we have $s\left(\tilde{a}_{1}\right)-s\left(\tilde{a}_{2}\right)<0$, i.e., $s\left(\tilde{a}_{1}\right)<s\left(\tilde{a}_{2}\right)$. Thus from Definition 1 , it follows that $\tilde{a}_{1}<\tilde{a}_{2}$, which completes the proof of Theorem 1 .

Deschrijver and Kerre [4] defined a complete lattice as a partially ordered set such that every nonempty subset of it have a supremum and an infimum, and defined a relation 
$\leq_{L^{*}}$ on $L^{*}=\{\tilde{a}=\langle[a, b],[c, d]\rangle \in D[0,1] \times D[0,1]:$ $b+d \leq 1\}$ as follows:

$$
\tilde{a}_{1} \leq_{L^{*}} \tilde{a}_{2} \Longleftrightarrow a_{1} \leq a_{2}, b_{1} \leq b_{2}, c_{1} \geq c_{2}, d_{1} \geq d_{2},
$$

and showed that $\left(L^{*}, \leq_{L^{*}}\right)$ is a complete lattice. However, in some situations, (7) cannot be used to compare IVIFNs. For example, let $\tilde{a}_{1}=\langle[0.2,0.4],[0.5,0.6]\rangle$ and $\tilde{a}_{2}=\langle[0.2,0.3],[0.4,0.7]\rangle$. Then it is impossible to know which one is bigger by using (7). But in this case, we use Definition 1 to compare them. In fact, since

$$
\begin{aligned}
& s\left(\tilde{a}_{1}\right)=\frac{1}{2}(0.2-0.5+0.4-0.6)=-0.25, \\
& s\left(\tilde{a}_{2}\right)=\frac{1}{2}(0.2-0.4+0.3-0.7)=-0.30,
\end{aligned}
$$

then, by Definition 1, we know that $\tilde{a}_{1}>\tilde{a}_{2}$.

Atanassov and Gargov [1] and Atanassov [2] introduced some basic operations on interval-valued intuitionistic fuzzy sets, which not only can ensure that the operational results are also interval-valued intuitionistic fuzzy sets but also are useful in the calculus of variables under interval-valued intuitionistic fuzzy environment. Motivated by the operations in [1] and [2], Xu [13] defined two operational laws of IVIFNs, which are very useful in the remainder of this paper, as follows:

Definition 2. Let $\tilde{a}_{1}=\left\langle\left[a_{1}, b_{1}\right],\left[c_{1}, d_{1}\right]\right\rangle, \tilde{a}_{2}=$ $\left\langle\left[a_{2}, b_{2}\right],\left[c_{2}, d_{2}\right]\right\rangle$ and $\tilde{a}=\langle[a, b],[c, d]\rangle$ be three IVIFNs; then

1) $\tilde{a}_{1} \otimes \tilde{a}_{2}=\left\langle\left[a_{1} a_{2}, b_{1} b_{2}\right],\left[c_{1}+c_{2}-c_{1} c_{2}, d_{1}+d_{2}-\right.\right.$ $\left.\left.d_{1} d_{2}\right]\right\rangle$

2) $\tilde{a}^{\lambda}=\left\langle\left[a^{\lambda}, b^{\lambda}\right],\left[1-(1-c)^{\lambda}, 1-(1-d)^{\lambda}\right]\right\rangle, \lambda>0$,

which can ensure the operational results are also IVIFNs. Based on Definition 2, Xu [13] further introduced the following relations:

1) $\tilde{a}_{1} \otimes \tilde{a}_{2}=\tilde{a}_{2} \otimes \tilde{a}_{1}$.

2) $\left(\tilde{a}_{1} \otimes \tilde{a}_{2}\right)^{\lambda}=\tilde{a}_{1}^{\lambda} \otimes \tilde{a}_{2}^{\lambda}, \lambda>0$.

3) $\tilde{a}^{\lambda_{1}} \otimes \tilde{a}^{\lambda_{2}}=\tilde{a}^{\lambda_{1}+\lambda_{2}}, \lambda_{1}, \lambda_{2}>0$.

Let $S\left(x_{1}, x_{2}\right)=x_{1}+x_{2}-x_{1} x_{2}$ and $T\left(y_{1}, y_{2}\right)=y_{1} y_{2}$ $(x=a, b ; y=c, d)$; then the operational law 1) in Definition 2 can be rewritten as follows:

$$
\begin{aligned}
& \tilde{a}_{1} \otimes \tilde{a}_{2} \\
& \quad=\left\langle\left[T\left(a_{1}, a_{2}\right), T\left(b_{1}, b_{2}\right)\right],\left[S\left(c_{1}, c_{2}\right), S\left(d_{1}, d_{2}\right)\right]\right\rangle,
\end{aligned}
$$

where $S\left(x_{1}, x_{2}\right)=x_{1}+x_{2}-x_{1} x_{2}$ and $T\left(y_{1}, y_{2}\right)=y_{1} y_{2}$ are, respectively, well-known $t$-conorm and $t$-norm satisfy boundedness, monotonicity, commutativity and associativity properties.

In the following, let us look at $\tilde{a}^{\lambda}$ for some special cases of $\lambda$ and $\tilde{a}$.

1) If $\tilde{a}=\langle[1,1],[0,0]\rangle$, then $\tilde{a}^{\lambda}=\langle[1,1],[0,0]\rangle$.
2) If $\tilde{a}=\langle[0,0],[1,1]\rangle$, then $\tilde{a}^{\lambda}=\langle[0,0],[1,1]\rangle$.

3) If $\tilde{a}=\langle[0,0],[0,0]\rangle$, then $\tilde{a}^{\lambda}=\langle[0,0],[0,0]\rangle$.

4) If $\tilde{a}=\langle[a, b],[c, d]\rangle, \lambda \rightarrow 0$ and $0<a \leq b<1$ and $0<c \leq d<1$, then $\tilde{a}^{\lambda} \rightarrow\langle[1,1],[0,0]\rangle$.

5) If $\tilde{a}=\langle[a, b],[c, d]\rangle, \lambda \rightarrow+\infty$ and $0<a \leq b<1$ and $0<c \leq d<1$, then $\tilde{a}^{\lambda} \rightarrow\langle[0,0],[1,1]\rangle$.

6) If $\tilde{a}=\langle[a, b],[c, d]\rangle$ and $\lambda=1$, then $\tilde{a}^{\lambda}=\tilde{a}$.

\subsection{Interval-valued Intuitionistic Fuzzy Geo- metric Aggregation Operators}

For convenience, let $\Omega$ be the set of all IVIFNs. Xu and Yager [16] introduced various geometric aggregation operators to deal with intuitionistic fuzzy information. Based on these operators and Definition 2, Xu [13] defined interval-valued intuitionistic fuzzy weighted geometric (IIFWG) operator:

Definition 3. [13] Let $\tilde{a}_{j}=\left\langle\left[a_{j}, b_{j}\right],\left[c_{j}, d_{j}\right]\right\rangle(j=$ $1,2, \ldots, n)$ be a collection of IVIFNs, and let IIFWG : $\Omega^{n} \rightarrow \Omega$, if

$\operatorname{IIFWG}_{w}\left(\tilde{a}_{1}, \tilde{a}_{2}, \ldots, \tilde{a}_{n}\right)=\tilde{a}_{1}^{w_{1}} \otimes \tilde{a}_{2}^{w_{2}} \otimes \cdots \otimes \tilde{a}_{n}^{w_{n}}$,

then IIFWG is called the IIFWG operator of dimension $n$, where $w=\left(w_{1}, w_{2}, \ldots, w_{n}\right)^{T}$ is the weight vector of $\tilde{a}_{j}$ $(j=1,2, \ldots, n)$ with $w_{j}>0$ and $\sum_{j=1}^{n} w_{j}=1$. Especially, if $w=\left(\frac{1}{n}, \frac{1}{n}, \ldots, \frac{1}{n}\right)^{T}$, then the IIFWG operator is reduced to the interval-valued intuitionistic fuzzy geometric (IIFG) operator, which is defined as follows:

$$
\operatorname{IIFG}_{w}\left(\tilde{a}_{1}, \tilde{a}_{2}, \ldots, \tilde{a}_{n}\right)=\left(\tilde{a}_{1} \otimes \tilde{a}_{2} \otimes \cdots \otimes \tilde{a}_{n}\right)^{\frac{1}{n}} .
$$

Based on Definition 3, we have the following.

Theorem 2. (Properties of IIFWG): Let $\tilde{a}_{j}=$ $\left\langle\left[a_{j}, b_{j}\right],\left[c_{j}, d_{j}\right]\right\rangle(j=1,2, \ldots, n)$ be a collection of IVIFNs and $w=\left(w_{1}, w_{2}, \ldots, w_{n}\right)^{T}$ is the weight vector of $\tilde{a}_{j}(j=1,2, \ldots, n)$ with $w_{j}>0$ and $\sum_{j=1}^{n} w_{j}=1$; then we have the following.

1) (Idempotency): If all $\tilde{a}_{j}=\left\langle\left[a_{j}, b_{j}\right],\left[c_{j}, d_{j}\right]\right\rangle(j=$ $1,2, \ldots, n)$ are equal, i.e., $\tilde{a}_{j}=\tilde{a}$ for all $j$, then

$$
\operatorname{IIFWG}_{w}\left(\tilde{a}_{1}, \tilde{a}_{2}, \ldots, \tilde{a}_{n}\right)=\tilde{a} .
$$

2) (Boundedness):

$\tilde{a}^{-} \leq \operatorname{IIFWG}_{w}\left(\tilde{a}_{1}, \tilde{a}_{2}, \ldots, \tilde{a}_{n}\right) \leq \tilde{a}^{+}$, for every $w(11)$ where $\tilde{a}^{-}=\left\langle\left[\min _{j} a_{j}, \min _{j} b_{j}\right],\left[\max _{j} c_{j}, \max _{j} d_{j}\right]\right\rangle$ and $\tilde{a}^{+}=\left\langle\left[\max _{j} a_{j}, \max _{j} b_{j}\right],\left[\min _{j} c_{j}, \min _{j} d_{j}\right]\right\rangle$.

3) (Monotonicity): Let $\tilde{a}_{j}^{*}=\left\langle\left[a_{j}^{*}, b_{j}^{*}\right],\left[c_{j}^{*}, d_{j}^{*}\right]\right\rangle(j=$ $1,2, \ldots, n)$ be a collection of IVIFNs. If $a_{j} \leq a_{j}^{*}, b_{j} \leq b_{j}^{*}$, $c_{j} \geq c_{j}^{*}$ and $d_{j} \geq d_{j}^{*}$ for all $j$, then

$\operatorname{IIFWG}_{w}\left(\tilde{a}_{1}, \tilde{a}_{2}, \ldots, \tilde{a}_{n}\right) \leq \operatorname{IIFWG}\left(\tilde{a}_{1}^{*}, \tilde{a}_{2}^{*}, \ldots, \tilde{a}_{n}^{*}\right)$.

Based on Definitions 1 and 2, Xu and Chen [14] defined another geometric operator for aggregating interval-valued intuitionistic fuzzy information. 
Definition 4. [14] Let $\tilde{a}_{j}=\left\langle\left[a_{j}, b_{j}\right],\left[c_{j}, d_{j}\right]\right\rangle(j=$ $1,2, \ldots, n)$ be a collection of IVIFNs. An interval-valued intuitionistic fuzzy ordered weighted geometric (IIFOWG) operator of dimension $n$ is a mapping IIFOWG : $\Omega^{n} \rightarrow \Omega$, that has an associated vector $\omega=\left(\omega_{1}, \omega_{2}, \ldots, \omega_{n}\right)^{T}$ such that $\omega_{j}>0$ and $\sum_{j=1}^{n} \omega_{j}=1$. Furthermore,

$$
\begin{aligned}
& \operatorname{IIFOWG}_{\omega}\left(\tilde{a}_{1}, \tilde{a}_{2}, \ldots, \tilde{a}_{n}\right) \\
& =\tilde{a}_{\sigma(1)}^{\omega_{1}} \otimes \tilde{a}_{\sigma(2)}^{\omega_{2}} \otimes \cdots \otimes \tilde{a}_{\sigma(n)}^{\omega_{n}},
\end{aligned}
$$

where $(\sigma(1), \sigma(2), \ldots, \sigma(n))$ is a permutation of $(1,2, \ldots, n)$ such that $\tilde{a}_{\sigma(j-1)} \geq \tilde{a}_{\sigma(j)}$ for all $j$. Especially, if $\omega=\left(\frac{1}{n}, \frac{1}{n}, \ldots, \frac{1}{n}\right)^{T}$, then the IIFWG operator is reduced to the IIFG operator.

Based on Definition 4, we have the following properties similar to those of the IIFWG operator.

Theorem 3. (Properties of IIFOWG): Let $\tilde{a}_{j}=$ $\left\langle\left[a_{j}, b_{j}\right],\left[c_{j}, d_{j}\right]\right\rangle(j=1,2, \ldots, n)$ be a collection of IVIFNs and $\omega=\left(\omega_{1}, \omega_{2}, \ldots, \omega_{n}\right)^{T}$ is the weighting vector of the IIFOWG operator, with $\omega_{j}>0$ and $\sum_{j=1}^{n} \omega_{j}=1$; then we have the following.

1) (Idempotency): If all $\tilde{a}_{j}=\left\langle\left[a_{j}, b_{j}\right],\left[c_{j}, d_{j}\right]\right\rangle(j=$ $1,2, \ldots, n)$ are equal, i.e., $\tilde{a}_{j}=\tilde{a}$ for all $j$, then

$$
\operatorname{IIFOWG} \omega\left(\tilde{a}_{1}, \tilde{a}_{2}, \ldots, \tilde{a}_{n}\right)=\tilde{a} .
$$

2) (Boundedness):

$\tilde{a}^{-} \leq \operatorname{IIFOWG}{ }_{\omega}\left(\tilde{a}_{1}, \tilde{a}_{2}, \ldots, \tilde{a}_{n}\right) \leq \tilde{a}^{+}$, for every $\omega$

where $\tilde{a}^{-}=\left\langle\left[\min _{j} a_{j}, \min _{j} b_{j}\right],\left[\max _{j} c_{j}, \max _{j} d_{j}\right]\right\rangle$ and $\tilde{a}^{+}=\left\langle\left[\max _{j} a_{j}, \max _{j} b_{j}\right],\left[\min _{j} c_{j}, \min _{j} d_{j}\right]\right\rangle$.

3) (Monotonicity): Let $\tilde{a}_{j}^{*}=\left\langle\left[a_{j}^{*}, b_{j}^{*}\right],\left[c_{j}^{*}, d_{j}^{*}\right]\right\rangle(j=$ $1,2, \ldots, n)$ be a collection of IVIFNs. If $a_{j} \leq a_{j}^{*}, b_{j} \leq b_{j}^{*}$, $c_{j} \geq c_{j}^{*}$ and $d_{j} \geq d_{j}^{*}$ for all $j$, then

$$
\begin{aligned}
& \operatorname{IIFOWG}_{\omega}\left(\tilde{a}_{1}, \tilde{a}_{2}, \ldots, \tilde{a}_{n}\right) \\
& \quad \leq \operatorname{IIFOWG} \omega\left(\tilde{a}_{1}^{*}, \tilde{a}_{2}^{*}, \ldots, \tilde{a}_{n}^{*}\right), \text { for every } \omega .
\end{aligned}
$$

4) (Commutativity): Let $\tilde{a}_{j}^{\prime}=\left\langle\left[a_{j}^{\prime}, b_{j}^{\prime}\right],\left[c_{j}^{\prime}, d_{j}^{\prime}\right]\right\rangle(j=$ $1,2, \ldots, n)$ be a collection of IVIFNs; then

$$
\begin{aligned}
& \operatorname{IIFOWG}_{\omega}\left(\tilde{a}_{1}, \tilde{a}_{2}, \ldots, \tilde{a}_{n}\right) \\
& \quad=\operatorname{IIFOWG}\left(\tilde{a}_{1}^{\prime}, \tilde{a}_{2}^{\prime}, \ldots, \tilde{a}_{n}^{\prime}\right), \text { for every } \omega
\end{aligned}
$$

where $\left(\tilde{a}_{1}^{\prime}, \tilde{a}_{2}^{\prime}, \ldots, \tilde{a}_{n}^{\prime}\right)$ is any permutation of $\left(\tilde{a}_{1}, \tilde{a}_{2}, \ldots\right.$, $\left.\tilde{a}_{n}\right)$.

Besides the above properties, the IIOWG operator has the following desirable results.

Theorem 4. Let $\tilde{a}_{j}=\left\langle\left[a_{j}, b_{j}\right],\left[c_{j}, d_{j}\right]\right\rangle(j=$ $1,2, \ldots, n)$ be a collection of IVIFNs and $\omega=$ $\left(\omega_{1}, \omega_{2}, \ldots, \omega_{n}\right)^{T}$ is the weighting vector of the IIFOWG operator, with $\omega_{j}>0$ and $\sum_{j=1}^{n} \omega_{j}=1$; then we have

1) If $\omega=(1,0, \ldots, 0)^{T}$, then

$$
\operatorname{IIFOWG}_{\omega}\left(\tilde{a}_{1}, \tilde{a}_{2}, \ldots, \tilde{a}_{n}\right)=\max _{j}\left(\tilde{a}_{j}\right) .
$$

2) If $\omega=(0,0, \ldots, 1)^{T}$, then

$$
\operatorname{IIFOWG}_{\omega}\left(\tilde{a}_{1}, \tilde{a}_{2}, \ldots, \tilde{a}_{n}\right)=\min _{j}\left(\tilde{a}_{j}\right) .
$$

3) If $\omega_{j}=1$ and $\omega_{i}=0(i \neq j)$, then

$$
\operatorname{IIFOWG}_{\omega}\left(\tilde{a}_{1}, \tilde{a}_{2}, \ldots, \tilde{a}_{n}\right)=\tilde{a}_{\sigma(j)},
$$

where $\tilde{a}_{\sigma(j)}$ is the $j$ th largest of $\tilde{a}_{i}(i=1,2, \ldots, n)$.

Consider that the IIFWG operator weights only the IVIFNs, while the IIFOWG operator weights only the ordered positions of IVIFNs instead of weighting the IVIFNs themselves. To overcome this limitation, $\mathrm{Xu}$ and Chen [14] defined an interval-valued intuitionistic fuzzy hybrid geometric (IIFHG) operator, which weights both the given IVIFN and its ordered position.

Definition 5. [14] An interval-valued intuitionistic fuzzy hybrid geometric (IIFHG) operator is a mapping IIFHG : $\Omega^{n} \rightarrow \Omega$, which has an associated vector $\alpha=$ $\left(\alpha_{1}, \alpha_{2}, \ldots, \alpha_{n}\right)^{T}$ with $\alpha_{j}>0$ and $\sum_{j=1}^{n} \alpha_{j}=1$, such that

$$
\begin{aligned}
\operatorname{IIFHG}_{\alpha, w} & \left(\tilde{a}_{1}, \tilde{a}_{2}, \ldots, \tilde{a}_{n}\right) \\
& =\dot{\tilde{a}}_{\sigma(1)}^{\alpha_{1}} \otimes \dot{\tilde{a}}_{\sigma(2)}^{\alpha_{2}} \otimes \cdots \otimes \dot{\tilde{a}}_{\sigma(n)}^{\alpha_{n}},
\end{aligned}
$$

where $\dot{\tilde{a}}_{\sigma(j)}$ is the $j$ th largest of the weighted IVIFNs $\dot{\tilde{a}}_{\sigma(j)}\left(\dot{\tilde{a}}_{\sigma(j)}=\tilde{a}_{j}^{n w_{j}}, j=1,2, \ldots, n\right), w=$ $\left(w_{1}, w_{2}, \ldots, w_{n}\right)^{T}$ is weight vector of $\tilde{a}_{j}(j=1,2, \ldots, n)$ with $w_{j}>0$ and $\sum_{j=1}^{n} w_{j}=1$, and $n$ is the balancing coefficient, which plays a role of balance (in this case, if the vector $w=\left(w_{1}, w_{2}, \ldots, w_{n}\right)^{T}$ approaches $\left(\frac{1}{n}, \frac{1}{n}, \ldots, \frac{1}{n}\right)^{T}$, then the vector $\left(\tilde{a}_{1}^{n w_{1}}, \tilde{a}_{2}^{n w_{2}}, \ldots, \tilde{a}_{n}^{n w_{n}}\right)^{T}$ approaches $\left.\left(\tilde{a}_{1}, \tilde{a}_{2}, \ldots, \tilde{a}_{n}\right)^{T}\right)$.

Especially, $w=\left(\frac{1}{n}, \frac{1}{n}, \ldots, \frac{1}{n}\right)^{T}$, then the IIFHG operator is reduced to the IIFWG operator; if $\alpha=$ $\left(\frac{1}{n}, \frac{1}{n}, \ldots, \frac{1}{n}\right)^{T}$, then the IIFHG operator is reduced to the IIFOWG operator.

\section{Multi-person Multi-attribute Decision Making under Interval-valued Intuitionistic Fuzzy Environment}

In this section, we investigate the group decision making problems in which all information provided by decision-makers is expressed as interval-valued intuitionistic fuzzy decision matrices where each of the elements is characterized by IVIFN, and the information about attribute weights is partially known.

For multi-person multi-attribute decision making problem, let $O=\left\{O_{1}, O_{2}, \ldots, O_{n}\right\}$ be the set of $n$ alternatives, $D=\left\{d_{1}, d_{2}, \ldots, d_{l}\right\}$ be the set of $l$ decision-makers, and $\lambda=\left(\lambda_{1}, \lambda_{2}, \ldots, \lambda_{l}\right)^{T}$ be the weight vector of decisionmakers, where $\lambda_{k} \geq 0, k=1,2, \ldots, l$, and $\sum_{k=1}^{l} \lambda_{k}=1$. 
Let $U=\left\{u_{1}, u_{2}, \ldots, u_{m}\right\}$ be the set of $m$ attributes. In general, the decision-makers need to determine the importance degrees of a set $U$ of $m$ attributes. Thus we suppose that the decision-makers provide the attribute weight information may be presented in the following forms [7, 6], for $i \neq j$ :

1. A weak ranking: $\left\{w_{i} \geq w_{j}\right\}$;

2. A strict ranking: $\left\{w_{i}-w_{j} \geq \delta_{i}(>0)\right\}$;

3. A ranking with multiples: $\left\{w_{i} \geq \delta_{i} w_{j}\right\}, 0 \leq \delta_{i} \leq 1$;

4. An interval form: $\left\{\delta_{i} \leq w_{i} \leq \delta_{i}+\epsilon_{i}\right\}, 0 \leq \delta_{i} \leq$ $\delta_{i}+\epsilon_{i} \leq 1$

5. A ranking of differences: $\left\{w_{i}-w_{j} \geq w_{k}-w_{l}\right\}$, for $j \neq k \neq l$.

For convenience, we denote by $H$ the set of the known information about attribute weights provided by the decisionmakers. Let $R^{(k)}=\left(\tilde{r}_{i j}^{(k)}\right)_{m \times n}$ be an intervalvalued intuitionistic fuzzy decision matrix, where $\tilde{r}_{i j}^{(k)}=$ $\left\langle\left[a_{i j}^{(k)}, b_{i j}^{(k)}\right],\left[c_{i j}^{(k)}, d_{i j}^{(k)}\right]\right\rangle$ is an IVIFN, provided by the decision-maker $d_{k} \in D$ for the alternative $O_{j}$ with respect to the attribute $u_{i} \in U,\left[a_{i j}^{(k)}, b_{i j}^{(k)}\right]$ indicates the degree that the alternative $O_{j} \in O$ satisfy the attribute $u_{i}$, expressed by the decision-maker $d_{k}$, while $\left[c_{i j}^{(k)}, d_{i j}^{(k)}\right]$ indicates the degree that the alternative $O_{j} \in O$ does not satisfy the attribute $u_{i}$, expressed by the decision-maker $d_{k}$, and

$$
\begin{aligned}
& {\left[a_{i j}^{(k)}, b_{i j}^{(k)}\right] \subset[0,1],\left[c_{i j}^{(k)}, d_{i j}^{(k)}\right] \subset[0,1],} \\
& \quad b_{i j}^{(k)}+d_{i j}^{(k)} \leq 1, i=1,2, \ldots, m, j=1,2, \ldots, n .
\end{aligned}
$$

To make a final decision in the process of group decision making, we need to fuse all individual decision opinion into group opinion. To do this, we use the IIFHG operator (see, (18)) to aggregate all individual interval-valued intuitionistic fuzzy decision matrices $R^{(k)}=\left(\tilde{r}_{i j}^{(k)}\right)_{m \times n}$ $(k=1,2, \ldots, l)$ into the collective interval-valued intuitionistic fuzzy decision matrix $R=\left(\tilde{r}_{i j}\right)_{m \times n}$, where

$$
\begin{aligned}
\tilde{r}_{i j}= & \operatorname{IIFHG}_{\alpha, \lambda}\left(\tilde{r}_{i j}^{(1)}, \tilde{r}_{i j}^{(2)}, \ldots, \tilde{r}_{i j}^{(l)}\right) \\
= & \left(\dot{\tilde{r}}_{i j}^{(\sigma(1))}\right)^{\alpha_{1}} \otimes\left(\dot{\tilde{r}}_{i j}^{(\sigma(2))}\right)^{\alpha_{2}} \otimes \cdots\left(\dot{\tilde{r}}_{i j}^{(\sigma(l))}\right)^{\alpha_{l}} \\
& =\left\langle\left[\prod_{k=1}^{n}\left(\dot{a}_{i j}^{(\sigma(k))}\right)^{\alpha_{k}}, \prod_{k=1}^{n}\left(\dot{b}_{i j}^{(\sigma(k))}\right)^{\alpha_{k}}\right],\right. \\
& {\left.\left[1-\prod_{k=1}^{n}\left(1-\dot{c}_{i j}^{(\sigma(k))}\right)^{\alpha_{k}}, 1-\prod_{k=1}^{n}\left(1-\dot{d}_{i j}^{(\sigma(k))}\right)^{\alpha_{k}}\right]\right\rangle, }
\end{aligned}
$$

where $\alpha=\left(\alpha_{1}, \alpha_{2}, \ldots, \alpha_{l}\right)^{T}$ is weight vector of IIFHG operator with $\alpha_{k}>0(k=1,2, \ldots, l)$ and $\sum_{k=1}^{l} \alpha_{k}=1$, and $\dot{\tilde{r}}_{i j}^{(\sigma(k))}=\left\langle\left[\dot{a}_{i j}^{\sigma((k))}, \dot{b}_{i j}^{\sigma((k))}\right],\left[\dot{c}_{i j}^{\sigma((k))}, \dot{d}_{i j}^{\sigma((k))}\right]\right\rangle$ is the $k$ th largest of the weighted IVIFNs $\dot{\tilde{r}}_{i j}^{(k)}\left(\dot{\tilde{r}}_{i j}^{(k)}=\left(\tilde{r}_{i j}^{(k)}\right)^{l \lambda_{k}}\right.$, $i=1,2, \ldots, m ; j=1,2, \ldots, n)$. Here, we denote by $\tilde{r}_{i j}=\left\langle\left[a_{i j}, b_{i j}\right],\left[c_{i j}, d_{i j}\right]\right\rangle, i=1,2, \ldots, m ; j=$ $1,2, \ldots, n$
In the cases that the information about attribute weights is completely known, that is, the weight vector $w=$ $\left(w_{1}, w_{2}, \ldots, w_{m}\right)^{T}$ of the attributes $u_{k}(k=1,2 \ldots, m)$ can be completely determine in advance, then based on the collective interval-valued intuitionistic fuzzy decision matrix $R=\left(\tilde{r}_{i j}\right)_{m \times n}$, we can use the IIFWG operator (see, (9)):

$$
\begin{aligned}
\tilde{r}_{j}= & \operatorname{IIFWG}_{w}\left(\tilde{r}_{1 j}, \tilde{r}_{2 j}, \ldots, \tilde{r}_{m j}\right) \\
& =\tilde{r}_{i j}^{w_{1}} \otimes \tilde{r}_{2 j}^{w_{2}} \otimes \cdots \otimes \tilde{r}_{m j}^{w_{m}} \\
& =\left\langle\left[\prod_{i=1}^{m} a_{i j}^{w_{i}}, \prod_{i=1}^{m} b_{i j}^{w_{1}}\right],\left[1-\prod_{i=1}^{m}\left(1-c_{i j}\right)^{w_{i}},\right.\right. \\
& \left.\left.1-\prod_{i=1}^{m}\left(1-d_{i j}\right)^{w_{i}}\right]\right\rangle, j=1,2, \ldots, n
\end{aligned}
$$

to obtain the overall values of the alternative $O_{j}$. The greater the value of $\tilde{r}_{j}$, the better the alternative $O_{j}$ will be.

However, the information about attribute weights provided by the decision-makers is usually incomplete (see, $[6,7])$. So an interesting and important issue is how to utilize the collective interval-valued intuitionistic fuzzy decision matrix and the known weight information to find the most desirable alternative(s).

In the following, we present an approach to determining the weight of attributes.

Definition 6. Let $R=\left(\tilde{r}_{i j}\right)_{m \times n}$ be the collective interval-valued intuitionistic fuzzy decision matrix. Then we call $S=\left(s_{i j}\right)_{m \times n}$ the score matrix of $R=\left(\tilde{r}_{i j}\right)_{m \times n}$, where

$$
\begin{gathered}
s_{i j}=s\left(\tilde{r}_{i j}\right)=\frac{1}{2}\left(a_{i j}-c_{i j}+b_{i j}-d_{i j}\right), \\
i=1,2, \ldots, m ; j=1,2, \ldots, n
\end{gathered}
$$

and $s\left(\tilde{r}_{i j}\right)$ is called the score of $\tilde{r}_{i j}$.

Based on the score matrix , we present the overall score values of each alternatives $O_{j}(j=1,2, \ldots, m)$ :

$$
s_{j}(w)=\sum_{i=1}^{m} w_{i} s_{i j}, j=1,2, \ldots, n .
$$

Obviously, the greater the value $s_{j}(w)$, the better the alternative $O_{j}$. When we only consider the alternative $O_{j}$, then a reasonable vector of attribute weights $w=$ $\left(w_{1}, w_{2}, \ldots, w_{m}\right)^{T}$ should be determined. Thus, we establish the following optimization model to maximize $s_{j}(w)$ :

$$
\begin{aligned}
& \text { Maximize } s_{j}(w)=\sum_{i=1}^{m} w_{i} s_{i j} \\
& \text { Subject to }: w=\left(w_{1}, \ldots, w_{m}\right)^{T} \in H, w_{i} \geq 0, \\
& i=1, \ldots, m, \sum_{i=1}^{m} w_{i}=1 .
\end{aligned}
$$


By solving the model (M), we obtain the optimal solution $w^{(j)}=\left(w_{1}^{(j)}, w_{2}^{(j)}, \ldots, w_{m}^{(j)}\right)^{T}$ corresponding to the alternative $O_{j}$. However, in the process of determining the weight vector $w=\left(w_{1}, w_{2}, \ldots, w_{m}\right)^{T}$, we need to consider all the alternatives $O_{j}(j=1,2, \ldots, n)$ as a whole. Thus, we construct weight matrix $W=\left(w_{i}^{(j)}\right)_{m \times n}$ of the optimal solutions $w^{(j)}=\left(w_{1}^{(j)}, w_{2}^{(j)}, \ldots, w_{m}^{(j)}\right)^{T}$ $(j=1,2, \ldots, n)$ as:

$$
W=\left(\begin{array}{cccc}
w_{1}^{(1)} & w_{1}^{(2)} & \cdots & w_{1}^{(n)} \\
w_{2}^{(1)} & w_{2}^{(2)} & \cdots & w_{2}^{(n)} \\
\vdots & \vdots & \vdots & \vdots \\
w_{m}^{(1)} & w_{m}^{(2)} & \cdots & w_{m}^{(n)}
\end{array}\right)
$$

and we calculate the normalized eigenvector $\omega=$ $\left(\omega_{1}, \omega_{2}, \ldots, \omega_{n}\right)^{T}$ of the matrix $\left(S^{T} W\right)^{T}\left(S^{T} W\right)$, and then we construct a combined weight vector as follows:

$$
\begin{aligned}
w & =W \omega=\left(\begin{array}{cccc}
w_{1}^{(1)} & w_{1}^{(2)} & \cdots & w_{1}^{(n)} \\
w_{2}^{(1)} & w_{2}^{(2)} & \cdots & w_{2}^{(n)} \\
\vdots & \vdots & \vdots & \vdots \\
w_{m}^{(1)} & w_{m}^{(2)} & \cdots & w_{m}^{(n)}
\end{array}\right)\left(\begin{array}{c}
\omega_{1} \\
\omega_{2} \\
\vdots \\
\omega_{n}
\end{array}\right) \\
& =\omega_{1} w^{(1)}+\omega_{2} w^{(2)}+\cdots+\omega_{n} w^{(n)}
\end{aligned}
$$

and thus we derive the weight vector $w=\left(w_{1}\right.$ $\left.w_{2}, \ldots, w_{m}\right)^{T}$ of the attributes $u_{k}(k=1,2 \ldots, m)$.

Based on the analysis above, in the following we present an approach to multi-person multi-attribute interval-valued intuitionistic fuzzy decision making with incomplete attribute weight information:

Step 1. Utilize the IIFHG operator (20) to aggregate all individual interval-valued intuitionistic fuzzy decision matrices $R^{(k)}=\left(\tilde{r}_{i j}^{(k)}\right)_{m \times n}(k=1,2, \ldots, l)$ into a collective interval-valued intuitionistic fuzzy decision matrix $R=\left(\tilde{r}_{i j}\right)_{m \times n}$.

Step 2. Calculate the score matrix $S=\left(s_{i j}\right)_{m \times n}$ of the collective interval-valued intuitionistic fuzzy decision matrix $R$.

Step 3. Utilize the model (M) to obtain the optimal weight vectors $w^{(j)}=\left(w_{1}^{(j)}, w_{2}^{(j)}, \ldots, w_{m}^{(j)}\right)^{T}(j=$ $1,2, \ldots, n)$ corresponding to the alternatives $O_{j}(j=$ $1,2, \ldots, n)$, and then construct the weight matrix $W$.

Step 4. Calculate the normalized eigenvector $\omega=$ $\left(\omega_{1}, \omega_{2}, \ldots, \omega_{n}\right)^{T}$ of the matrix $\left(S^{T} W\right)^{T}\left(S^{T} W\right)$.

Step 5. Utilize (24) to derive the weight vector $w=$ $\left(w_{1}, w_{2}, \ldots, w_{m}\right)^{T}$.

Step 6. Use the IIFWG operator (21) to get the overall values $\tilde{r}_{j}$ of the alternatives $O_{j}(j=1,2, \ldots, n)$.

Step 7. Use the score function to calculate the scores $s\left(\tilde{r}_{j}\right)$ of the overall values $\tilde{r}_{j}$ of the alternatives $O_{j}(j=$ $1,2, \ldots, n)$.
Step 8. Utilize the scores $s\left(\tilde{r}_{j}\right)$ to rank the alternatives $O_{j}(j=1,2, \ldots, n)$, and then select the most desirable one(s) (if two scores $s\left(\tilde{r}_{i}\right)$ and $s\left(\tilde{r}_{j}\right)$ are identical, then we calculate the accuracy degrees $h\left(\tilde{r}_{i}\right)$ and $h\left(\tilde{r}_{j}\right)$ of the overall values $\tilde{r}_{i}$ and $\tilde{r}_{j}$, respectively, and then rank the alternatives $O_{i}$ and $O_{j}$ according to the accuracy degrees $h\left(\tilde{r}_{i}\right)$ and $\left.h\left(\tilde{r}_{j}\right)\right)$.

\section{Numerical Example}

In this section, we present a numerical example to illustrate how the proposed method can be used.

Let $O=\left\{O_{1}, O_{2}, O_{3}\right\}$ be the set of three alternatives, $D=\left\{d_{1}, d_{2}, d_{3}, d_{4}\right\}$ be the set of four experts whose weight vector is $\lambda=(0.3,0.2,0.3,0.2)^{T}$. Let $U=\left\{u_{1}, u_{2}, u_{3}, u_{4}, u_{5}\right\}$ be the set of five attributes. The experts $d_{k}(k=1,2,3,4)$ represent, respectively, the characteristics of the alternatives $O_{j}(j=1,2,3)$ by the IVIFNs $\tilde{r}_{i j}^{(k)}(i=1,2,3,4,5 ; j=1,2,3)$ with respect to the attributes $u_{i}(i=1,2,3,4,5)$, listed in Tables 1-4 (i.e., interval-valued intuitionistic fuzzy decision matrices $\left.R^{(k)}=\left(\tilde{r}_{i j}^{(k)}\right)_{5 \times 3}(k=1,2,3,4)\right)$.

Table 1. Interval-valued intuitionistic fuzzy decision matrix $R^{(1)}$

\begin{tabular}{c|ccc}
\hline & $\mathrm{O}_{1}$ & $\mathrm{O}_{2}$ & $\mathrm{O}_{3}$ \\
\hline$u_{1}$ & $\langle[0.5,0.6],[0.2,0.3]\rangle$ & $\langle[0.3,0.4],[0.4,0.6]\rangle$ & $\langle[0.4,0.5],[0.3,0.5]\rangle$ \\
$u_{2}$ & $\langle[0.3,0.5],[0.4,0.5]\rangle$ & $\langle[0.1,0.3],[0.2,0.4]\rangle$ & $\langle[0.7,0.8],[0.1,0.2]\rangle$ \\
$u_{3}$ & $\langle[0.6,0.7],[0.2,0.3]\rangle$ & $\langle[0.3,0.4],[0.4,0.5]\rangle$ & $\langle[0.5,0.8],[0.1,0.2]\rangle$ \\
$u_{4}$ & $\langle[0.5,0.7],[0.1,0.2]\rangle$ & $\langle[0.2,0.4],[0.5,0.6]\rangle$ & $\langle[0.4,0.6],[0.2,0.3]\rangle$ \\
$u_{5}$ & $\langle[0.1,0.4],[0.3,0.5]\rangle$ & $\langle[0.7,0.8],[0.1,0.2]\rangle$ & $\langle[0.5,0.6],[0.2,0.3]\rangle$ \\
\hline
\end{tabular}

Table 2. Interval-valued intuitionistic fuzzy decision matrix $R^{(2)}$

\begin{tabular}{c|ccc}
\hline & $O_{1}$ & $O_{2}$ & $O_{3}$ \\
\hline$u_{1}$ & $\langle[0.4,0.5],[0.2,0.4]\rangle$ & $\langle[0.3,0.5],[0.4,0.5]\rangle$ & $\langle[0.4,0.6],[0.3,0.4]\rangle$ \\
$u_{2}$ & $\langle[0.3,0.4],[0.4,0.6]\rangle$ & $\langle[0.1,0.3],[0.3,0.7]\rangle$ & $\langle[0.6,0.8],[0.1,0.2]\rangle$ \\
$u_{3}$ & $\langle[0.6,0.7],[0.1,0.2]\rangle$ & $\langle[0.3,0.4],[0.4,0.5]\rangle$ & $\langle[0.7,0.8],[0.1,0.2]\rangle$ \\
$u_{4}$ & $\langle[0.5,0.6],[0.1,0.3]\rangle$ & $\langle[0.2,0.3],[0.6,0.7]\rangle$ & $\langle[0.4,0.6],[0.3,0.4]\rangle$ \\
$u_{5}$ & $\langle[0.1,0.3],[0.3,0.5]\rangle$ & $\langle[0.6,0.8],[0.1,0.2]\rangle$ & $\langle[0.5,0.6],[0.2,0.4]\rangle$ \\
\hline
\end{tabular}

Table 3. Interval-valued intuitionistic fuzzy decision matrix $R^{(3)}$

\begin{tabular}{c|ccc}
\hline & $O_{1}$ & $O_{2}$ & $O_{3}$ \\
\hline$u_{1}$ & $\langle[0.4,0.7],[0.1,0.2]\rangle$, & $\langle[0.4,0.5],[0.2,0.4]\rangle$ & $\langle[0.2,0.4],[0.3,0.4]\rangle$ \\
$u_{2}$ & $\langle[0.3,0.5],[0.3,0.4]\rangle$ & $\langle[0.2,0.4],[0.4,0.5]\rangle$ & $\langle[0.6,0.8],[0.1,0.2]\rangle$ \\
$u_{3}$ & $\langle[0.6,0.7],[0.1,0.2]\rangle$ & $\langle[0.4,0.5],[0.3,0.4]\rangle$ & $\langle[0.5,0.7],[0.1,0.3]\rangle$ \\
$u_{4}$ & $\langle[0.5,0.6],[0.1,0.3]\rangle$ & $\langle[0.1,0.2],[0.7,0.8]\rangle$ & $\langle[0.5,0.7],[0.2,0.3]\rangle$ \\
$u_{5}$ & $\langle[0.3,0.5],[0.4,0.5]\rangle$ & $\langle[0.6,0.7],[0.2,0.3]\rangle$ & $\langle[0.6,0.8],[0.1,0.2]\rangle$ \\
\hline
\end{tabular}

Table 4. Interval-valued intuitionistic fuzzy decision matrix $R^{(4)}$

\begin{tabular}{c|ccc}
\hline & $O_{1}$ & $O_{2}$ & $O_{3}$ \\
\hline$u_{1}$ & $\langle[0.6,0.7],[0.2,0.3]\rangle$ & $\langle[0.4,0.5],[0.4,0.5]\rangle$ & $\langle[0.4,0.5],[0.3,0.4]\rangle$ \\
$u_{2}$ & $\langle[0.3,0.4],[0.3,0.4]\rangle$ & $\langle[0.1,0.2],[0.2,0.3]\rangle$ & $\langle[0.6,0.7],[0.1,0.3]\rangle$ \\
$u_{3}$ & $\langle[0.7,0.8],[0.1,0.2]\rangle$ & $\langle[0.3,0.4],[0.5,0.6]\rangle$ & $\langle[0.5,0.8],[0.1,0.2]\rangle$ \\
$u_{4}$ & $\langle[0.5,0.6],[0.1,0.3]\rangle$ & $\langle[0.2,0.3],[0.4,0.6]\rangle$ & $\langle[0.4,0.5],[0.2,0.3]\rangle$ \\
$u_{5}$ & $\langle[0.1,0.2],[0.5,0.7]\rangle$ & $\langle[0.6,0.7],[0.1,0.2]\rangle$ & $\langle[0.5,0.6],[0.3,0.4]\rangle$ \\
\hline
\end{tabular}


Assume that the information about attribute weights, given by decision-makers, is shown as follows, respectively:

$$
\begin{aligned}
& d_{1}: w_{1} \leq 0.3,0.2 \leq w_{3} \leq 0.5 \\
& d_{2}: 0.1 \leq w_{2} \leq 0.2, w_{5} \leq 0.4 \\
& d_{3}: w_{3}-w_{2} \geq w_{5}-w_{4}, w_{4} \geq w_{1} \\
& d_{4}: w_{3}-w_{1} \leq 0.1,0.1 \leq w_{4} \leq 0.3
\end{aligned}
$$

Then the set $H$ of the known information about attribute weights provided by the decision-makers is

$$
H=\left\{w_{1} \leq 0.3,0.2 \leq w_{3} \leq 0.5,0.1 \leq w_{2} \leq 0.2,\right.
$$

$$
\begin{aligned}
& w_{5} \leq 0.4, w_{3}-w_{2} \geq w_{5}-w_{4}, w_{4} \geq w_{1}, \\
& \left.w_{3}-w_{1} \leq 0.1,0.1 \leq w_{4} \leq 0.3\right\}
\end{aligned}
$$

Step 1. Utilize the IIFHG operator (let $\alpha=(0.155$, $0.345,0.345,0.155)^{T}$ be its weight vector derived by the normal distribution based method [12]) to aggregate the individual interval-valued intuitionistic fuzzy decision matrices $R^{(k)}=\left(\tilde{r}_{i j}^{(k)}\right)_{5 \times 3}(k=1,2,3,4)$ into the collective interval-valued intuitionistic fuzzy decision matrix $R=\left(\tilde{r}_{i j}\right)_{5 \times 3}$ (Table 5).

Table 5. Collective interval-valued intuitionistic fuzzy decision matrix $R$

\begin{tabular}{l|ccc}
\hline & $O_{1}$ & $O_{2}$ & $O_{3}$ \\
\hline$u_{1}$ & $\langle[0.4385,0.6199],[0.1549,0.2848]\rangle$ & $\langle[0.3502,0.4797],[0.3114,0.4681]\rangle$ & $\langle[0.3516,0.4906],[0.2940,0.4214]\rangle$ \\
$u_{2}$ & $\langle[0.3000,0.4573],[0.3404,0.4710]\rangle$ & $\langle[0.1138,0.3010],[0.2511,0.4773]\rangle$ & $\langle[0.6395,0.7711],[0.0980,0.2263]\rangle$ \\
$u_{3}$ & $\langle[0.6116,0.7117],[0.1089,0.2083]\rangle$ & $\langle[0.3379,0.4387],[0.3872,0.4887]\rangle$ & $\langle[0.5213,0.7804],[0.0980,0.2083]\rangle$ \\
$u_{4}$ & $\langle[0.5000,0.6395],[0.0980,0.2567]\rangle$ & $\langle[0.1758,0.3134],[0.5305,0.6496]\rangle$ & $\langle[0.4387,0.6252],[0.2263,0.3262]\rangle$ \\
$u_{5}$ & $\langle[0.1323,0.3623],[0.3747,0.5482]\rangle$ & $\langle[0.6395,0.7521],[0.1089,0.2083]\rangle$ & $\langle[0.5452,0.6502],[0.1770,0.3005]\rangle$ \\
\hline
\end{tabular}

Step 2. Calculate the score matrix $S=\left(s_{i j}\right)_{5 \times 3}$ of the collective interval-valued intuitionistic fuzzy decision matrix $R$ (Table 6):

Table 6. Collective score matrix $S$

\begin{tabular}{c|ccc}
\hline & $O_{1}$ & $O_{2}$ & $O_{3}$ \\
\hline$u_{1}$ & 0.3093 & 0.0252 & 0.0634 \\
$u_{2}$ & -0.0270 & -0.1568 & 0.5431 \\
$u_{3}$ & 0.5030 & -0.0496 & 0.4977 \\
$u_{4}$ & 0.3924 & -0.3454 & 0.2557 \\
$u_{5}$ & -0.2141 & 0.5372 & 0.3589 \\
\hline
\end{tabular}

Step 3. Use the method (M-1) to obtain the optimal weight vectors $w^{(j)}=\left(w_{1}^{(j)}, w_{2}^{(j)}, w_{3}^{(j)}, w_{4}^{(j)}, w_{5}^{(j)}\right)^{T}(j=$ $1,2,3)$ corresponding to the alternatives $O_{j}(j=1,2,3)$

$$
\begin{aligned}
& w^{(1)}=(0.2667,0.1,0.3667,0.2667,0)^{T}, \\
& w^{(2)}=(0.16,0.1,0.26,0.16,0.32)^{T}, \\
& w^{(3)}=(0.1,0.2,0.2,0.25,0.25)^{T}
\end{aligned}
$$

and construct the weight matrix

$$
W=\left(\begin{array}{lll}
0.2667 & 0.16 & 0.1 \\
0.1 & 0.1 & 0.2 \\
0.3667 & 0.26 & 0.2 \\
0.2667 & 0.16 & 0.25 \\
0 & 0.32 & 0.25
\end{array}\right)
$$

then

$$
\left(S^{T} W\right)^{T}\left(S^{T} W\right)=\left(\begin{array}{lll}
0.2539 & 0.1650 & 0.1804 \\
0.1650 & 0.1602 & 0.1589 \\
0.1804 & 0.1589 & 0.1648
\end{array}\right) .
$$

Step 4. Calculate the normalized eigenvectors $\omega$ of the matrix $\left(S^{T} W\right)^{T}\left(S^{T} W\right)$ :

$$
\omega=(0.3814,0.3025,0.3162)^{T} \text {. }
$$

Step 5. Use (24) to derive the weight vector $w$ :

$$
\begin{aligned}
w & =W \omega=\left(\begin{array}{lll}
0.2667 & 0.16 & 0.1 \\
0.1 & 0.1 & 0.2 \\
0.3667 & 0.26 & 0.2 \\
0.2667 & 0.16 & 0.25 \\
0 & 0.32 & 0.25
\end{array}\right)\left(\begin{array}{l}
0.3814 \\
0.3025 \\
0.3162
\end{array}\right) \\
& =(0.1817,0.1316,0.2817,0.2292,0.1758)^{T} .
\end{aligned}
$$

Step 6. Use the IIFWG operator to obtain the overall values $\tilde{r}_{j}(j=1,2,3)$ of the alternatives $O_{j}(j=1,2,3)$ :

$$
\begin{aligned}
& \tilde{r}_{1}=\langle[0.3824,0.5675],[0.2007,0.3417]\rangle, \\
& \tilde{r}_{2}=\langle[0.2838,0.4319],[0.3542,0.4885]\rangle, \\
& \tilde{r}_{3}=\langle[0.4830,0.6592],[0.1804,0.2969]\rangle .
\end{aligned}
$$

Step 7. Use the score function (3) to calculate the score $s\left(\tilde{r}_{j}\right)(j=1,2,3)$ of the overall values $\tilde{r}_{j}(j=1,2,3)$ of the alternatives $O_{j}(j=1,2,3)$ :

$$
s\left(\tilde{r}_{1}\right)=0.2037, s\left(\tilde{r}_{2}\right)=-0.0635, s\left(\tilde{r}_{3}\right)=0.3324 .
$$

and thus,

$$
s\left(\tilde{r}_{3}\right)>s\left(\tilde{r}_{1}\right)>s\left(\tilde{r}_{2}\right) .
$$

Step 8. Use the scores $s\left(\tilde{r}_{j}\right)(j=1,2,3)$ to rank the alternatives $O_{j}(j=1,2,3)$ :

$$
\mathrm{O}_{3}>\mathrm{O}_{1}>\mathrm{O}_{2}
$$

and then the most desirable alternative is $\mathrm{O}_{3}$. 


\section{Conclusions}

Based on the IIFHG and IIFWG operators, we have investigated the multi-person multi-attribute decision making problems under interval-valued intuitionistic fuzzy environment, and developed an approach to handling the situations where the attribute values are characterized by IVIFNs, and the information about attribute weights is partially known. In future, we shall continue working in the application of the IIFHG and IIFWG operators to other domains.

\section{References}

[1] K. Atanassov and G. Gargov, "Interval-valued intuitionistic fuzzy sets," Fuzzy Sets Sys., vol. 31, pp. 343349, 1989.

[2] K. Atanassov, "Operators over interval-valued intuitionistic fuzzy sets," Fuzzy Sets Sys., vol. 64, pp. 159174, 1994.

[3] H. Bustince and P. Burillo, "Correlation of intervalvalued intuitionistic fuzzy sets," Fuzzy Sets Sys., vol. 74, pp. 237-244, 1995.

[4] G. Deschrijver and E.E. Kerre, "On the relationship betwe en some extensions of fuzzy set theory," Fuzzy Sets Sys., vol. 133, pp. 227-235, 2003.

[5] D.H. Hong, "A note on correlation of interval-valued intuitionistic fuzzy sets," Fuzzy Sets Sys., vol. 95, pp. 113-117, 1998.

[6] S.H. Kim and B.S. Ahn, "Interactive group decision making procedure under incomplete information," European J. Operational Research, vol. 116, pp. 498-507, 1999.

[7] S.H. Kim, S.H. Choi and H. Kim, "An interactive procedurefor multiple attribute group decision making with incomplete information: Range-based approach," European J. Operational Research, vol. 118, pp. 139-152, 1999.

[8] X.D. Li, S.H. Zheng and F.L. Xiong, "Entropy and subsethood for general interval-valued intuitionistic fuzzy sets," LNCS, vol. 3613, pp. 42-52, 2005.

[9] T.K. Mondal and S.K. Samanta, "Topology of interval-valued intuitionistic fuzzy sets," Fuzzy Sets Sys., vol. 119, pp. 483-494, 2001.

[10] M.K. Sayadi, M. Heydari and K. Shahanaghi, "Extension of VIKOR method for decision making problem with interval numbers," Appl. Math. Modelling, in press, 2008.
[11] G.W. Wei and X.R. Wang, "Some geometric aggregation operators on interval-valued intuitionistic fuzzy sets and their appication to group decision making," Proc. 2007 ICCIS, pp. 495-499, 2007.

[12] Z.S. Xu, "An overview of methods for determining OWA weights," Internat. J. Intelligent Systems, vol. 20, pp. 843-865, 2005.

[13] Z.S. Xu, "Methods for aggregating interval-valued intuitionistic fuzzy information and their application to decision making," Control and Decision, vol. 22, pp. 215-219, 2007.

[14] Z.S. Xu and J. Chen, "On geometric aggregation over interval-valued intuitionistic fuzzy information," Proc. Fourth International Conference on Fuzzy Systems and Knowledge Discovery (FSKD 2007), vol. 2, pp. 466-471, 2007.

[15] Z.S. Xu and J. Chen, "An approach to group decision making based on interval-valued intuitionistic judgement matrices," System Engineer-Theory \& Practice, vol. 27, pp. 126-133, 2007.

[16] Z.S. Xu and R.R. Yager, "Some geometric aggregation operators based on intuitionistic fuzzy sets," Internat. J. General Systems, vol. 35, pp. 417-433, 2006.

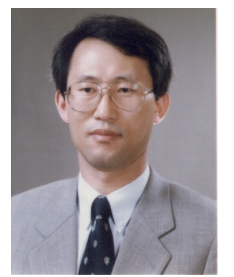

Jin Han Park received the B.S. degree, M.S. degree, and Ph.D. degree from the Department of Mathematics, Dong-A University, Korea, in the field of general topology. He is currently a Professor of the Department of Applied Mathematics, Pukyong National University, Korea. His research interests include general topology, fuzzy mathematics, decision making. E-mail : jihpark@pknu.ac.kr

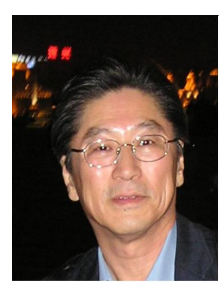

Young Chel Kwun received the B.S. and M.S. degree in mathematics from Dong-A University, Busan, Korea in 1981 and 1983, respectively and the Ph.D. degrees in applied mathematics from Dong-A University, Busan, Korea in 1990. He was a Visiting Scholar in the Department of applied mathematics, Kobe University, Kobe, Japan, from 2004 to 2005. He is currently a Professor in the Depart-ment of Mathematics, Dong-A University, Korea. His cur-rent research interests inclued fuzzy differential equations, informatics, mathematical theory, partial differential equations, system theory and control.

E-mail : yckwun@dau.ac.kr 
Multi-person Multi-attribute Decision Making Problems Based on Interval-valued Intuitionistic Fuzzy Information

Mi Jung Son received the B.S. degree, M.S. degree, and Ph.D. degree from the Department of Mathematics, DongA University, Korea, in the field of general topology. She is currently a Lecturer of the Department of Mathematics, Korea Maritime University, Korea. Her research interests include general topology, fuzzy mathematics, decision making.

E-mail : mjson72@korea.com 